\title{
Mass and Free Energy of Lovelock Black Holes
}

\author{
David Kastor $^{a 1}$, Sourya Ray ${ }^{b 2}$ and Jennie Traschen ${ }^{a 3}$ \\ ${ }^{a}$ Department of Physics, University of Massachusetts, Amherst, MA 01003 \\ ${ }^{b}$ Centro de Estudios Cientficos (CECS), Casilla 1469, Valdivia, Chile \\ ${ }^{1}$ kastor@physics.umass.edu , ${ }^{2}$ ray@cecs.cl, ${ }^{3}$ traschen@physics.umass.edu
}

\begin{abstract}
An explicit formula for the ADM mass of an asymptotically AdS black hole in a generic Lovelock gravity theory is presented, identical in form to that in Einstein gravity, but multiplied by a function of the Lovelock coupling constants and the AdS curvature radius. A Gauss' law type formula relates the mass, which is an integral at infinity, to an expression depending instead on the horizon radius. This and other thermodynamic quantities, such as the free energy, are then analyzed in the limits of small and large horizon radius, yielding results that are independent of the detailed choice of Lovelock couplings. In even dimensions, the temperature diverges in both limits, implying the existence of a minimum temperature for black holes. The negative free energy of sufficiently large black holes implies the existence of a Hawking-Page transition. In odd dimensions the temperature still diverges for large black holes, which again have negative free energy. However, the temperature vanishes as the horizon radius tends to zero and sufficiently small black holes have positive specific heat.
\end{abstract}




\section{Introduction}

Many interesting, stationary black holes are not known in analytic form. The list includes charged rotating black holes in $D>4$, black rings in $D>5$, localized Kaluza-Klein black holes and rotating black holes in Lovelock gravity theories. Results have been obtained using a variety of approximate techniques, including e.g. perturbative expansions in the slowly rotating limit [1, 2, 3, 4, 5] or effective field theory methods [6, 7, 8]. However, one may also ask whether in the absence of analytic solutions, it might still be possible to at least obtain certain properties of such spacetimes, such as thermodynamic ones, exactly.

In this paper we will address this question for a class of spacetimes that might best be called "semi-unknown", namely static Lovelock black holes. It is known [9] that these spacetimes are Schwarzschild-like, in the sense that the metric written in the general static, spherically symmetric form

$$
d s^{2}=-\phi(r) d t^{2}+\frac{d r^{2}}{f(r)}+r^{2} d \Omega_{D-2}^{2},
$$

additionally has $\phi(r)=f(r)$. For a generic Lovelock theory, the function $f(r)$ must satisfy an algebraic equation of order $\left[\frac{D-1}{2}\right]$, where $D$ is the spacetime dimension and the closed brackets denote taking the integer part. The coefficients of the polynomial are the coupling constants of the higher curvature terms in the Lovelock Lagrangian. Since the roots of a generic polynomial equation can be found in terms of radicals only up to order 4 , it follows that in spacetimes dimensions $D>10$ the general solution for a static Lovelock black hole cannot be written down in a simple closed form and hence remains unknown].

We make use of results from references [18, 19, 20], derived using the Hamiltonian formulation [21], to obtain exact properties of Lovelock black holes, without requiring the explicit (and in general unknown) form of the metric function $f(r)$. In fact with future applications to other, even more unknown, black holes in mind, we will "forget" that we know that $\phi(r)=f(r)$ and take the general static, spherically symmetric form (1) as our starting point. Our focus throughout will be on black holes with asymptotically AdS boundary conditions.

Further motivation comes from recent work on the CFT duals of asymptotically AdS solutions to higher curvature gravities. Significant work has been done, for example, on the relation between CFT plasmas and their gravitational duals, [22, 23, 24, 25] and on implications for the CFT of causality and stability in the bulk using holography [26, 27, 28, 29, 30, 31, 32, 33, 34, 35, 36, 37, 38, 39, 40]. As the correspondence between the higher

\footnotetext{
${ }^{1}$ Given that the solutions to generic cubic and quartic equations are quite cumbersome, in practice the general solution is only known in useful form for $D \leq 6$, although certain non-generic solutions such as those for Gauss-Bonnet gravity [10, 11, 12, 13, or Chern-Simons gravity [14, 15] are known in all dimensions. Note also that an analytic solution for a rotating black hole in $D=5$ Gauss-Bonnet gravity has also been found in the case of Chern-Simons couplings [16] (see also [17]).
} 
curvature bulk theory and the boundary CFT becomes better understood, it is interesting to ask whether the constructs we will make use of below, the Killing-Lovelock potentials and the associated Gauss' law relations that connect behavior at the horizon to behavior at infinity, have an analogue in the dual CFT.

The paper will proceed as follows. In section (2) we recall the basic elements of Lovelock gravity theories [41] that will subsequently be used. In section (3) we will derive the expression for the ADM mass of an asymptotically AdS Lovelock black hole in terms of its far field behavior. The ADM boundary integral receives contributions from each higher curvature term as well as from the Einstein term. The formula for the ADM mass is implicit in the work of [42] which computed the entropy in Lovelock theories and established the first law. However significant additional steps are required to make the result of [42] fully explicit. The expression we find ultimately takes the simple form of the standard ADM mass integral multiplied by a function of the Lovelock couplings and the asymptotic AdS curvature radius. This formula for the mass constitutes a new result.

In section (41) we review certain elements from our previous work [18, 19, 20] that we will be making use of. This includes the Killing-Lovelock potentials which allow for the derivation of a Gauss' law type expression for the Hamiltonian constraint, which will be our main tool. This expression relates certain boundary integrals at infinity to surface integrals on the horizon, without requiring the explicit form of the metric in between. For reference, we also present the Smarr formula [20] which gives the mass in terms of the entropy and additional thermodynamic quantities that arise in the Lovelock theory.

The bulk of our new results appear in sections (5) and (6). In section (5) we use the Gauss' law formula to obtain an expression for the mass, originally given by the far field behavior of the metric, in terms of the horizon radius of the black hole. With the goal in mind of providing a simple expression for the free energy, we also present formulas for the entropy, surface gravity and other thermodynamic quantities in terms of the horizon radius. The detailed behavior of these expressions over the full range of horizon radii depends on the choice of Lovelock coupling constants. However, we find that they have generic behavior in the limits of small and large horizon radii. There are qualitative differences between even and odd dimensions that arise in the small black hole regime. In particular, one finds that in odd dimensions there exists a "mass gap", in the sense that the mass tends to a finite value as the horizon radius tends to zero. The Hawking temperature also vanishes in this limit and sufficiently small black holes have positive specific heat. For even dimensions, however, the mass tends to zero for vanishing horizon radius and the specific heat for small black holes is negative.

In section (6) we assemble ingredients to construct a relatively simple expression for the free energy of black holes in generic Lovelock theories. The free energy had previously been computed only up to inclusion of the quadratic Gauss-Bonnet term [12, 43] in the Lovelock Lagrangian. The free energy for a stationary black hole solution is generally obtained 
by computing its Euclidean action. We emphasize that our method does not require the explicit functional form of the metric. We analyze the free energy and also the specific heat in the small and large black hole limits and comment upon the Hawking-Page phase transition for generic AdS-Lovelock black holes. In section (7) we briefly summarize our results and offer some directions for further related work.

\section{Lovelock gravity}

The Lagrangian of a Lovelock gravity theory in $D$ spacetime dimensions is given by

$$
\mathcal{L}=\frac{1}{16 \pi G} \sum_{k=0}^{k_{\max }} b_{k} \mathcal{L}^{(k)}
$$

where $k_{\max }=[(D-1) / 2]$ and the $b_{k}$ are real-valued coupling constants. The symbol $\mathcal{L}^{(k)}$ stands for the contraction of $k$ powers of the Riemann tensor given by

$$
\mathcal{L}^{(k)}=\frac{1}{2^{k}} \delta_{c_{1} d_{1} \ldots c_{k} d_{k}}^{a_{1} b_{1} \ldots a_{k} b_{k}} R_{a_{1} b_{1}}{ }^{c_{1} d_{1}} \ldots R_{a_{k} b_{k}}{ }^{c_{k} d_{k}} .
$$

where the $\delta$-symbol is the totally anti-symmetrized product normalized so that it takes nonzero values \pm 1 . The term $\mathcal{L}^{(0)}$ gives the cosmological constant term in the Lagrangian, while $\mathcal{L}^{(1)}$ gives the Einstein-Hilbert term and $\mathcal{L}^{(2)}$ the quadratic Gauss-Bonnet term. The upper bound in the sum (2) comes about because $\mathcal{L}^{(k)}$ vanishes identically for $D<2 k$ and turns out to make no contribution to the equations of motion in $D=2 k$.

The equations of motion for Lovelock gravity can be written as $\mathcal{G}^{a}{ }_{b}=0$ where

$$
\mathcal{G}^{a}{ }_{b}=\sum_{k=0}^{k_{\max }} b_{k} \mathcal{G}^{(k) a}{ }_{b} .
$$

We will not need the explicit expressions for these quantities. However, it is crucial in what follows that each of the tensors in this sum satisfies a conservation law $\nabla_{a} \mathcal{G}^{(k) a}{ }_{b}=0$.

Depending on the values of the coupling constants $b_{k}$ the theory may have anywhere from zero up to $k_{\max }$ distinct constant curvature vacuum solutions. Because we will be focusing on asymptotically AdS black holes below, our considerations will implicitly be limited to the subset of theories admitting at least one constant negative curvature vacuum. We will denote the curvature radius of this AdS vacuum by $l$.

We will also need the Hamiltonian formulation of Lovelock gravity which was developed in [21]. As usual in the Hamiltonian picture the spacetime metric is split according to

$$
g_{a b}=-n_{a} n_{b}+s_{a b}
$$


where $n^{a}$ is the unit timelike normal to a spatial slice $\Sigma$ with induced metric $s_{a b}$ and these satisfy the orthogonality relation $s_{a b} n^{b}=0$. As in Einstein gravity the time-time and time-space components of the field equations act as constraints on initial data. In Lovelock gravity the Hamiltonian and momentum constraint operators $H=-2 n^{a} n^{b} \mathcal{G}_{a b}$ and $H_{a}=-2 s_{a}^{b} n^{c} \mathcal{G}_{b c}$ are given by the sums

$$
H=\sum_{k=0}^{k_{\max }} b_{k} H^{(k)}, \quad H_{a}=\sum_{k=0}^{k_{\max }} b_{k} H_{a}^{(k)}
$$

with $H^{(k)}=-2 n^{a} n^{b} \mathcal{G}_{a b}^{(k)}$ and $H_{a}^{(k)}=-2 s_{a}{ }^{b} n^{c} \mathcal{G}_{b c}^{(k)}$. We will particularly need the expression for

$$
H^{(k)}=-\frac{1}{2^{k}} \tilde{\delta}_{c_{1} d_{1} \ldots c_{k} d_{k}}^{a_{1} b_{1} \ldots a_{k} b_{k}} R_{a_{1} b_{1}}{ }^{c_{1} d_{1}} \ldots R_{a_{k} b_{k}} c_{k} d_{k}
$$

where the tilde on the $\delta$-symbol indicates that its indices are projected with the spatial metric $s^{a}{ }_{b}$. The actual Hamiltonian function for evolution of initial data with respect to a vector field $\xi^{a}$ is then given by $\mathcal{H}_{\xi}=F H+F^{a} H_{a}$, where the lapse and shift $\left(F, F^{a}\right)$ are the components of the vector field $\xi^{a}$ normal to and along the spatial slice, so that $\xi^{a}=F n^{a}+F^{a}$. As in Einstein gravity the lapse and shift are Lagrange multipliers.

Finally, for use below we define the sums

$$
s_{(n)}=\sum_{k=0}^{k_{\max }}(-1)^{k+n} \frac{k ! \hat{b}_{k}}{(k-n) ! l^{2 k-2}}
$$

where the rescaled coefficients $\hat{b}_{k}$ are given by $\hat{b}_{k}=(D-1) ! b_{k} /(D-2 k-1) !$. Note that the combinations $\hat{b}_{k} / l^{2(k-1)}$ are dimensionless. The condition $s_{(0)}=0$, in fact, determines the allowed constant curvature vacua of the theory, while the sums $s_{(1)}$ and $s_{(2)}$ will turn up in our results below.

\section{ADM Mass}

An expression for the ADM mass of an asymptotically AdS Lovelock black hole can be obtained using the methods of Regge and Teitelboim [44]. We find a simple, explicit formula which is similar to that for the ADM mass. As in Einstein gravity, a boundary term must be included in the Lovelock Hamiltonian to ensure that the Hamiltonian variational principle correctly yields the equations of motion. The variation of this boundary term cancels another boundary term arising via integration by parts from the variation of the bulk Hamiltonian. The ADM mass is defined to be the value of the Hamiltonian divided by a factor of $16 \pi G$. Since the bulk Hamiltonian vanishes on solutions, the ADM mass is simply proportional to the value of the Hamiltonian boundary term. Implicit here is that 
the vector $\xi^{a}$ determining the direction of Hamiltonian evolution should be asymptotic to the time translation Killing vector of AdS.

This procedure was formally carried out in reference [42] as part of establishing the first law for Lovelock black holes. Each higher curvature term in the Lovelock theory makes a contribution to the Hamiltonian boundary term. For asymptotically flat solutions, possible if $b_{0}=0$ in the Lagrangian (2), because of the fall-off of the curvature tensor, only the boundary term corresponding to the Einstein term is nonzero at infinity. The formula for the mass is then the same as in Einstein gravity. However, the non-zero asymptotic value of the curvature tensor for AdS Lovelock black holes leads to contributions from all the higher curvature boundary terms. We will see, in fact, that the result is given by the usual ADM integral multiplied by a function of the Lovelock couplings and the background AdS curvature. To our knowledge this expression has not been derived in the literature 2 .

The Regge-Teitelboim type construction of the ADM mass in 42 yields an expression of the form

$$
M=-\frac{1}{16 \pi G} \sum_{k=0}^{k_{\max }} b_{k} \int_{\infty} d a_{c} B^{(k) c} .
$$

We will determine the boundary integrands $B^{(k) c}$ explicitly for asymptotically AdS boundary conditions. Because our interest is focused on static black holes, we will assume that the momentum and the shift vector vanish sufficiently rapidly in the asymptotic region that they do not play a role in this construction. It is then sufficient to retain only the term $\mathcal{H}_{\xi}=F H$ in the Hamiltonian and to take the curvature tensor in (7) to be that of the spatial metric $s_{a b}$. Now, assume that the spatial metric $s_{a b}$ solves the Lovelock constraint equations with asymptotically AdS boundary conditions and add to it an arbitrary perturbation $h_{a b}$ that also respects these boundary conditions. To first order, the perturbation to the Riemann tensor is then $\delta R_{a b}{ }^{c d}=R_{a b}{ }^{e[c} h_{e}{ }^{d]}-2 D_{[a} D^{[c} h_{b]}{ }^{d]}$, where $D_{a}$ is the covariant derivative operator for the spatial metric $s_{a b}$. Plugging this in to the variation of (7) yields the perturbation of the $k$ th Hamiltonian

$$
\delta H^{(k)}=-\frac{k}{2^{k}} \tilde{\delta}_{c_{1} d_{1} \ldots c_{k} d_{k}}^{a_{1} b_{1} \ldots a_{k} b_{k}} R_{a_{1} b_{1}}{ }^{c_{1} d_{1}} \ldots R_{a_{k-1} b_{k-1}}{ }^{c_{k-1} d_{k-1}}\left(R_{a_{k} b_{k}}{ }^{e c_{k}} h_{e}{ }^{d_{k}}-2 D_{a_{k}} D^{c_{k}} h_{b_{k}}{ }^{d_{k}}\right)
$$

\footnotetext{
${ }^{2} \mathrm{~A}$ number of related results have been established in the literature. The Hamiltonian approach of 44 has been used to obtain an expression for the mass in pure Lovelock gravity theories [15] with only a single term in Lagrangian. Significant work has also been done in the case of Gauss-Bonnet gravity. Deser and Tekin have extended the formalism of Abbott and Deser [45 to general quadratic theories of gravity [46, 47. The mass in Gauss-Bonnet gravity has also been found by means of a Noether's current construction by Deruelle et. al. 48 and using the Palatini formalism by Katz and Livshits 49 The mass of asymptotically AdS black holes in general higher curvature theories has also been addressed in [50.

Padilla [51] uses the Cartan formalism to derive a general expression for the mass in Gauss-Bonnet gravity by transforming the trace $K$ boundary term in the action into Hamiltonian variables. By assuming that a generalization of this result holds in general Lovelock theories, the mass of static Lovelock black hole is identified by Cai [52] with a constant of integration arising from integration of the Hamiltonian constraint. Our general derivation of the mass in Lovelock theories demonstrates the correctness of this assumption.
} 
Combining these, multiplying by the lapse function $F$, integrating by parts, and making use of the Bianchi identity for the Riemann tensor then gives an overall expression of the form

$$
F \delta H=\delta H^{*} \cdot F+\sum_{k} b_{k} D_{c} B^{(k) c} .
$$

Here the first term represents an adjoint differential operator acting on the lapse function $F$ and the vectors $B^{(k) c}$ are given by

$$
B^{(k) c}=\frac{k}{2^{k}} \tilde{\delta}_{a b e_{1} f_{1} \ldots e_{k} f_{k}}^{c d m_{1} n_{1} \ldots m_{k} n_{k}} R_{m_{1} n_{1}}^{e_{1} f_{1}} \ldots R_{m_{k} n_{k-1}}^{e_{k-1} f_{k-1}}\left(F D^{a} h_{d}^{b}-h_{d}^{b} D^{a} F\right)
$$

These are the vectors appearing in the expression for the ADM mass given above. The quantity $h_{a b}$ is then the deviation of the static black hole metric from the asymptotic AdS background, while the minus sign in (9) arises from the cancellation of boundary terms required in the Regge-Teitelboim construction. The expression can be made more fully explicit by noting that near infinity only the leading order background AdS curvature $R_{a b}{ }^{c d}=\left(1 / l^{2}\right) \delta_{a b}^{c d}$ contributes. This leads to the result

$$
M=\frac{s_{(1)}}{(D-1)(D-2)} \cdot\left(\frac{-1}{16 \pi G} \int_{\infty} d a_{c}\left\{F\left(D^{c} h-D_{b} h^{c b}\right)-h D^{c} F+h^{c b} D_{b} F\right\}\right) .
$$

The part of the formula in parenthesis is the usual expression for the ADM mass. The sum $s_{(1)}$, defined in (8), depends on the Lovelock coupling constants and the curvature radius $l$ of the asymptotic AdS vacuum. For Einstein gravity with a cosmological constant the prefactor outside the parenthesis reduces to one. The asymptotic form of the lapse function is $F=\sqrt{1+r^{2} / l^{2}}$, and therefore terms in (13) involving the derivative of the lapse can make non-trivial contributions to the mass. In the asymptotically flat case, these terms fall off too quickly to contribute and the integrand reduces to that of the ADM mass in the asymptotically flat case.

\section{Killing-Lovelock Potentials}

Killing-Lovelock potentials [18, 20] allow the Lovelock Hamiltonian constraint equations for a spacetime with a Killing vector to be written in a Gauss' law form. In reference [20] this property was used to derive an extended first law including variation of the Lovelock coupling constants and also a related Smarr formula for Lovelock black holes. This will also be our primary tool below.

For a spacetime, such as a stationary black hole, with a Killing vector $\xi^{a}$ the KillingLovelock potentials were defined in [18] to be antisymmetric tensors $\beta^{(k) a b}$ satisfying the relations

$$
\nabla_{a} \beta^{(k) a b}=-2 \mathcal{G}^{(k) a}{ }_{b} \xi^{b} .
$$


Their existence is guaranteed by the vanishing divergence of the right hand side. However, they are not uniquely determined, since a divergenceless tensor such as the divergence of an arbitrary 3-index antisymmetric tensor may always be added. This ambiguity does not affect results such as the extended first law or Smarr formula.

Now consider Hamiltonian evolution with respect to the Killing vector, so that the lapse and shift are given by the decomposition $\xi^{a}=F n^{a}+F^{a}$. The definition of the Killing-Lovelock potentials allows us to write the $k$ th Hamiltonian function as a total divergence,

$$
\mathcal{H}^{(k)}=F H^{(k)}+F^{a} H_{a}^{(k)}=-2 \mathcal{G}_{c}^{(k) d} \xi^{c} n_{d}=D_{c}\left(\beta^{(k) c d} n_{d}\right) .
$$

The Hamiltonian constraint equation $\mathcal{H}=0$ can then be written in the form of Gauss's law. It ultimately follows from this (see [20] for how this works) that the Killing-Lovelock potentials make contributions to the thermodynamics of AdS-Lovelock black holes that are similar in form to that of the electrostatic potential in the case of a charged black hole. For example, one contribution to the change in energy in the extended first law of [20] is proportional to the difference in the integrals of Killing potential over the boundaries of a spatial slice at infinity and at the black hole horizon. The AdS boundary conditions require a subtraction at infinity of the Killing-Lovelock potential $\beta_{A d S}^{(k) a b}$ for the asymptotic AdS spacetime resulting in thermodynamic potentials

$$
\Theta^{(k)}=-\left(\int_{\infty} d a r_{c}\left(\beta^{(k) c d}-\beta_{A d S}^{(k) c d}\right) n_{d}-\int_{h} d a r_{c} \beta^{(k) c d} n_{d}\right)
$$

that multiply variations $\delta b_{k}$ of the Lovelock couplings in the extended first law. As a consequence of the constraint equations these thermodynamic potentials satisfy the sum rule

$$
\sum_{k=0}^{k_{\max }} b_{k} \Theta^{(k)}=0
$$

The Smarr formula relates the mass of a black hole which is defined in terms of the behavior of the metric near infinity to the entropy which comes from the behavior at the horizon. For static Lovelock black holes the Smarr formula [20], which we will also make use of below, is given by

$$
\left((D-3)+2 \frac{s_{(2)}}{s_{(1)}}\right) M=\frac{\kappa}{2 \pi}\left[(D-2) S-S^{\prime}\right]-\Theta
$$

The entropy $S$ [42] has contributions from the higher curvature Lovelock terms and is given by $S=\hat{A} / 4 G$ with $\hat{A}=\sum_{k} b_{k} A_{k}$ and

$$
A_{k}=k \int_{h} d^{D-2} x \sqrt{\gamma} \mathcal{L}^{(k-1)}\left(\gamma_{a b}\right)
$$


where $\gamma_{a b}$ is the induced metric on the boundary of the spatial slice at the horizon. There is also an additional entropy-like contribution at the horizon as well as the net contribution Lovelock thermodynamic potentials which are given respectively by

$$
S^{\prime}=\frac{1}{2 G} \sum_{k=0}^{k_{\max }}(k-1) b_{k} A^{(k)}, \quad \Theta \equiv \frac{1}{8 \pi G} \sum_{k=0}^{k_{\max }}(k-1) b_{k} \Theta^{(k)} .
$$

The Smarr formula is derived in [20] via an overall scaling argument from an extended form of the first law in which variations of the dimensionful Lovelock coupling constants are taken into account. The contribution of $S^{\prime}$ to the Smarr formula arises from the explicit dependence of the entropy on the Lovelock couplings, and the second term in the parenthesis on the left in (18) similarly arises from the dependence of the mass (9) on the couplings $b_{k}$.

For Einstein gravity with vanishing cosmological constant one finds that $s_{(2)}=S^{\prime}=\Theta=0$ and one recovers Smarr formula for static, asymptotically flat black holes. The case of Einstein gravity with non-zero cosmological constant was discussed in [19]. In this case, one still has $s_{(2)}=S^{\prime}=0$. However, there is a non-trivial contribution to $\Theta$ from the product $3 \Theta^{(0)}$. In [19] it was argued that $\Theta^{(0)}$ can be regarded as minus an effective volume behind the black hole horizon. Since the cosmological constant is proportional to minus the background pressure, one sees that the $k=0$ term in the Smarr formula has the form $p V$ which is familiar from classical thermodynamics. The mass $M$ should then be regarded as the spacetime enthalpy. This interpretation was explored further in [53, 54].

\section{$5 \quad$ Lovelock black holes}

In this section we will derive formulas for the basic thermodynamic properties of static, spherically symmetric AdS Lovelock black holes in terms of the horizon radius $r_{h}$ and the Lovelock parameters $b_{k}$. We consider static, spherical symmetric, asymptotically AdS spacetimes with AdS curvature radius $l$. The metric can then be taken to have the form (1), where at large radius the metric functions have the asymptotic forms

$$
\phi(r) \sim 1+\frac{r^{2}}{l^{2}}-\frac{c_{t}}{r^{D-3}}, \quad f(r) \sim 1+\frac{r^{2}}{l^{2}}-\frac{c_{r}}{r^{D-3}}
$$

for some constants $c_{t}$ and $c_{r}$. We assume that there is a Killing horizon at $r=r_{h}$, where $\phi\left(r_{h}\right)=0$. Static spherically symmetric black hole solutions of Lovelock gravity theories have been known for some time, starting with the work of [10, 11] in the Gauss-Bonnet case and [9] in the general Lovelock case (see also [55]). These solutions all have $\phi(r)=f(r)$ and the field equations reduce to the requirement that $f(r)$ solve a certain polynomial

\footnotetext{
${ }^{3}$ One has $\Lambda=-2 b_{0}$ in this case.
} 
equation of order $k_{\max }$, with coefficients determined by the Lovelock coupling constant 4 . Except in certain special cases, such as Einstein or Gauss-Bonnet gravity or when the Lovelock couplings are tuned such that the polynomial has a unique degenerate root [15], the solutions for $f(r)$ are generally not known explicitly.

Here we will use the general relations given in the previous sections to derive expressions for the mass $M$, surface gravity $\kappa$, entropy $S$ and free energy $F$ of Lovelock black holes in terms of $r_{h}$ without requiring the explicit solution of the field equation. This approach provides a geometrical understanding of the formulae. As noted in the introduction, with the application to other even less explicitly known black hole spacetimes in mind, we will take the general form of the metric (1) as our starting point and "forget" that we know that solutions to the field equations will have $\phi(r)=f(r)$.

\subsection{Constant curvature vacua and Killing potentials}

Working in the Hamiltonian picture, we assume that for a spherically symmetric static black hole the spatial metric has the form

$$
s_{a b} d x^{a} d x^{b}=\frac{d r^{2}}{f(r)}+r^{2} d \Omega_{D-2}^{2},
$$

that the extrinsic curvature $K_{a b}$ vanishes, and that the Hamiltonian evolution is carried out along the static Killing field $\xi^{a}=F n^{a}$. The function $F^{2}$ must then have the same large $r$ fall off conditions as the metric function $\phi$ in (21) and satisfy $F^{2}\left(r_{h}\right)=0$. Substituting the spatial metric into the Lovelock Hamiltonian functions $H^{(k)}$ in (7) we find that we find for these metrics that

$$
H^{(k)}=-\gamma_{k} \frac{1}{r^{(D-2)}} \frac{\partial}{\partial r}\left(r^{(D-1-2 k)}(1-f)^{k}\right)
$$

where $\gamma_{k}=\frac{(D-2) !}{(D-2 k-1) !}$. The constraint equation is

$$
H=\sum_{k=0}^{k_{\max }} b_{k} H^{(k)}=0
$$

First consider the background AdS metric with radius of curvature $l$ and no black hole. The AdS functions are $F_{A d S}^{2}=f_{A d S}=1+r^{2} / l^{2}$. Since the metric satisfies the constraint equation with $f_{A d S}$, substituting into (24) gives a relation between the couplings and $l^{2}$,

$$
\sum_{k=0}^{k_{\max }}(-1)^{k} \frac{\hat{b}_{k}}{l^{2(k-1)}}=0
$$

\footnotetext{
${ }^{4}$ Specifically, if one defines the function $F$ according to $f=1-r^{2} F$, then $F$ must satisfy the polynomial equation $\sum_{k=0}^{k_{\max }} \hat{b}_{k} F^{k}=\frac{\omega}{r^{D-1}}$ for some constant $\omega$. Constant curvature vacua of Lovelock theories solve this equation with $\omega=0$.
} 
As noted above, this is the condition that the sum $s_{(0)}$ defined above in (8) vanish in a constant curvature vacuum. Whether, or not, there are real positive solutions for $l^{2}$ depends on the values of the Lovelock couplings. We will assume that we are working with a Lovelock gravity theory that has at least one real, positive solution $l^{2}$.

Next we find the projected Killing-Lovelock potentials $\beta^{(k) r d} n_{d}$, which are solutions to equation (15) with vanishing shift vector $F^{a}$. Plugging in the form of the $k$ th Hamiltonian (23) this becomes

$$
\sqrt{f} \frac{\partial}{\partial r}\left(\frac{r^{(D-2)}}{\sqrt{f}} \beta^{(k) r d} n_{d}\right)=-F \gamma_{k} \frac{\partial}{\partial r}\left(r^{(D-1-2 k)}(1-f)^{k}\right)
$$

Now, recall that the existence of the Killing-Lovelock potentials depends on the the existence of a Killing vector $\xi^{a}$, but not on the metric being a solution to the field equations. The statement that the thermodynamic potentials $\Theta^{(k)}$ sum to zero in (17), however, does assume that the constraint equation is satisfied. Since we will want to make use of (17) our current task is to solve for the Killing-Lovelock potentials assuming that $f$ solves the constraint equation (24). Note also that the differential equation (26) includes a factor of the lapse function $F$. In the Hamiltonian picture the lapse is a gauge choice that may be freely specified 5 . We can choose any function $F$ consistent with $-F^{2}$ being the norm of the static Killing field for a spherically symmetric AdS black hole. Specifically, we can choose any $F$ that depends only on $r$ with $F\left(r_{h}\right)=0$ and satisfying the large $r$ fall off conditions in (21).

On the other hand, inspection shows that equation (26) is easy to solve if we can choose $F=\sqrt{f}$. This choice has the correct fall off conditions at large $r$, since the function $f(r)$ does. Therefore, we only need to worry about the condition that $F$ vanishes at the horizon, or equivalently whether the function $f(r)$ necessarily vanishes at the horizon? Of course it is well known that the Schwarzchild solution in Einstein gravity has $F=\sqrt{f}$, with $f(r)$ vanishing at the horizon. As noted above the static Lovelock black holes continue to have this Schwarzchild form [9]. However, one can also demonstrate that $f\left(r_{h}\right)=0$ under weaker conditions than requiring that the full set of field equations be solved. Let us assume that the surface gravity for the black hole metric (1) is finite and non-zero, and that the constraint equation is satisfied at the horizon radius $r_{h}$. We show in the Appendix that these conditions imply that $f \sim F^{2}$ near the horizon, and therefore that $f\left(r_{h}\right)=0$. Hence we can choose $F=\sqrt{f}$ in (26) and the solution for the projected Killing potentials are

$$
\beta^{(k) r d} n_{d}=-\gamma_{k} r^{1-2 k} \sqrt{f}(1-f)^{k}
$$

In the next section we make use of these solutions to express the mass in terms of the horizon radius $r_{h}$.

\footnotetext{
${ }^{5}$ Note that if one arbitrarily specifies $F$ at each subsequent time in the evolution, then in general the evolution will not be along a Killing field. To keep the evolution along the Killing vector, one imposes $\dot{s}_{a b}=\dot{K}_{a b}=0$, which implies a set of differential equations that $F$ must satisfy.
} 


\subsection{Dependence of mass on horizon radius}

It is straightforward to evaluate the boundary integral for the ADM mass (13) in terms of the coefficient $c_{r}$ that characterizes the far field behavior of the spatial metric. With the AdS boundary conditions (21), one finds that the terms in (13) that depend on the derivative of the lapse $F$ cancel, and the integral becomes

$$
M=\frac{\Omega_{D-2} s_{(1)} c_{r}}{16 \pi G(D-1)},
$$

where we have taken $b_{1}=1$. We next turn to the task of finding the fall-off coefficient $c_{r}$ and hence also the mass in terms of the horizon radius $r_{h}$ by applying the results (16) and (17). The integrand at large $r$ in (16) becomes

$$
r^{(D-2 k-1)}\left[(1-f)^{k}-\left(1-f_{A d S}\right)^{k}\right] \simeq(-1)^{k-1} \frac{k c_{r}}{l^{2(k-1)}}
$$

while the boundary term on the horizon is easily evaluated using the condition $f\left(r_{h}\right)=0$. Assembling these pieces one arrives at the expression for the thermodynamic potentials

$$
\Theta^{(k)}=-\gamma_{k} \Omega_{D-2}\left(r_{h}^{D-2 k-1}+(-1)^{k} \frac{k c_{r}}{l^{2(k-1)}}\right)
$$

where $\Omega_{D-2}$ is the area of a unit $D-2$ sphere. For $k=0$ this reproduces the result of [19] that $\Theta^{(0)}$ is given by minus an effective volume of the region behind the horizon. Finally, requiring that the sum rule (17) be satisfied yields the relation

$$
c_{r}=\left(\frac{r_{h}^{D-3}}{s_{(1)}}\right) \sum_{k=0}^{k_{\max }} \frac{\hat{b}_{k}}{r_{h}^{2(k-1)}}
$$

This is a key result since it relates the far field behavior of the black hole solution to the horizon radius, without making use of an exact analytic expression for $f(r)$ in the region between.

There are two important points to be made about the expression for $c_{r}$. First, we have noted that the solutions for static Lovelock black holes are known to be specified in terms of solutions to a polynomial equation of order $k_{\max }$ in the function $f(r)$ [9. This equation arises from integrating the Hamiltonian constraint and $c_{r}$ is a constant of integration. Equation (31) may also be obtained by evaluating this polynomial at $r_{h}$ [52. What we have learned from our more general treatment is that the relation (31) expresses the fact that the Hamiltonian constraint is a total divergence when defined with respect to evolution along a Killing field. Put differently, if a metric does not have a Killing field we have no reason to expect that the mass is simply a function of data on the horizon, but in general will depend on volume integrals as well. 
Second, we can ask whether there a computational advantage to working with the Loveloock potentials and the sum rule (17) compared to simply writing out the field equations and analyzing them? In the spherically symmetric case this is likely a matter of taste. The analysis in terms of the Killing-Lovelock potentials is more complicated, but exposes an underlying geometrical structure. For more complicated spacetimes, such as rotating black holes, it may be that using the Killing potentials allows one to find geometrical relations of interest more simply than through a brute force analysis of the field equations.

We can now substitute in for $c_{r}$ using (31) and obtain the sought after, result for the mass of a static Lovelock black hole in terms of its horizon radius

$$
M=\frac{\Omega_{D-2} r_{h}^{D-3}}{16 \pi G(D-1)} \sum_{k} \frac{\hat{b}_{k}}{r_{h}^{2(k-1)}} .
$$

This generalizes to all Lovelock theories the result for Gauss-Bonnet black holes given in [12. Such a generalization was assumed to hold in [52] and we have now shown that this is indeed the case.

To get an idea of how this formula behaves, let us examine the dependence of the mass on $r_{h}$ in different limiting regimes. The behavior of the mass as $r_{h} \rightarrow 0$ turns out to be interesting in that it differs between even and odd spacetime dimensions. Since the small black holes are dominated by the highest curvature terms, behavior in this limit depends on whether the order $k_{\max }$ term actually appears in the Lagrangian with non-zero coupling constant. We will assume that this is the case. Note that this includes Gauss-Bonnet gravity in $D=5$ and in $D=6$, for which $k_{\max }=2$, but not in higher dimension.

By a "small" black hole will be one such that the horizon radius is sufficiently small that $r_{h}^{2\left(k_{\max }-k\right)} \ll\left|\hat{b}_{k_{\max }} / \hat{b}_{k}\right|$ for all $k<k_{\max }$. After noting that $k_{\max }=(D-1) / 2$, and $2 k_{\max }=$ $(D-2) / 2$ respectively in odd and even dimensions, one finds that in the small black hole regime the mass depends on $r_{h}$ as

$$
\begin{array}{cc}
M \approx \frac{\Omega_{D-2}}{16 \pi G(D-1)} \hat{b}_{k_{\max }} r_{h} & D \text { even } \\
M \approx \frac{\Omega_{D-2}}{16 \pi G(D-1)}\left(\hat{b}_{k_{\max }}+\hat{b}_{k_{\max }-1} r_{h}^{2}\right) & D \text { odd }
\end{array}
$$

However, for $D$ odd the mass goes to a nonzero value, We see that for even dimensions, the mass goes smoothly to zero with the horizon radius as it does for Schwarzschild black holes. However, for odd dimensions there is a minimum mass for black holes that is proportional to $\hat{b}_{k_{\max }}$. This minimum mass, or mass gap, has been discussed previously for $D=5$ GaussBonnet black holes in [12] and for Chern-Simons-Lovelock theories, which have a unique constant curvature vacuum, in [15].

So long as the coefficient $b_{0}$ of the cosmological constant term in the Lovelock Lagrangian is non-zero, black holes in the opposite regime of large $r_{h}$ are dominated by the cosmological 
constant and look qualitatively the same in all dimensions. A "large" large black hole will be one such that the horizon radius satisfies $r_{h}^{2 k} \gg\left|\hat{b}_{k} / \hat{b}_{0}\right|$ for all $k>0$. For large black holes one finds that the mass depends on the horizon radius as

$$
M \approx \frac{\Omega_{D-2}}{16 \pi G(D-1)} b_{0} r_{h}^{D-1} .
$$

\subsection{Surface gravity of Lovelock black holes}

For a metric of the general form (1) the surface gravity is given by $\kappa=(1 / 2) \phi^{\prime}\left(r_{h}\right)$. In the appendix we argue that near the horizon of a black hole the metric functions must satisfy $g_{t t} \approx-1 / g_{r r}$, with equality for the functions and their first derivatives at the horizon. Hence $f\left(r_{h}\right)=0$ and $f^{\prime}\left(r_{h}\right)=2 \kappa$. Evaluating the Hamiltonian function $H^{(k)}$ in (23) at the horizon and applying the sum rule (17) yields a general relation between the surface gravity and the horizon radius without the need for the explicit form of the metric function $f(r)$,

$$
\kappa\left(\sum_{k=0}^{k_{\max }} \frac{k \hat{b}_{k}}{r_{h}^{2(k-1)}}\right)=\frac{1}{2 r_{h}} \sum_{k=0}^{k_{\max }} \frac{(D-2 k-1) \hat{b}_{k}}{r_{h}^{2(k-1)}}
$$

For Gauss-Bonnet gravity this agrees with the expressions for the surface gravity obtained from the explicit solutions in [12, 43, 52] using an approach similar to the one here.

Let us examine at the behavior of the surface gravity in various limits regimes starting with small black holes as defined above. In this regime, one finds that

$$
\begin{array}{cc}
\kappa \approx \frac{1}{2 k_{\max } r_{h}}, & \text { D even } \\
\kappa \approx \frac{\hat{b}_{k_{\max } r_{h}}}{k_{\max } \hat{b}_{\left(k_{\max }-1\right)}}, & \text { D odd }
\end{array}
$$

Again, we see a qualitative difference between even and odd dimensions. In even dimensions the surface gravity diverges in the limit of vanishing horizon radius, as it does for Schwarzschild black holes in $D=4$. However for generic Lovelock theories in odd dimensions, i.e. those in which $b_{k_{\max }}$ is nonvanishing, the surface gravity goes smoothly to zero with the horizon radius. This change in behavior was noted for Gauss Bonnet gravity in $D=5,6$ in [12]. Here we note, in agreement with the observations of [52], that this is characteristic of Lovelock black holes in general.

On the other hand, the surface gravity of large black holes has qualitatively the same behavior for all AdS-Lovelock black holes. One finds that

$$
\kappa \approx \frac{b_{0} r_{h}}{2(D-2)}
$$

where we have set $b_{1}=1$, so that $\hat{b}_{1}=(D-1)(D-2)$. In any dimension, therefore, there exists a large black hole solution for sufficiently high temperature. In even dimensions also 
always exists a small black hole at suficiently high temperatures. Hence for $D$ even, and so long as the Lovelock couplings are such that the surface gravity stays positive for all horizon radii, there will be a minimum temperature at which static black holes exist, while in odd dimensions there is no minimum temperature. In addition, there may be local extrema of the temperature depending on the choices of the $b_{k}$.

\subsection{Entropy, thermodynamic potential and Smarr relation}

The expression for the entropy [42] given in section (4) is a sum of integrals of Lovelock invariants constructed from the induced metric $\gamma_{a b}$ on the horizon cross section. For a spherically symmetric black hole $\gamma_{a b}$ is the metric of a round $D-2$ dimensional sphere of radius $r_{h}$ and the Riemann tensor is given simply by $R_{a b}{ }^{c d}=\left(1 / r_{h}^{2}\right) \delta_{a b}^{c d}$. Evaluating the various Lovelock terms explicitly gives $\mathcal{L}^{(k-1)}\left(\gamma_{a b}\right)=\frac{(D-2) !}{(D-2 k) !} r_{h}^{-2(k-1)}$. The entropy for a static, spherically symmetric Lovelock black hole can then be written as

$$
S=\frac{\Omega_{D-2} r_{h}^{D-2}}{4(D-1) G} \sum_{k=0}^{k_{\max }} \frac{k \hat{b}_{k}}{(D-2 k) r_{h}^{2(k-1)}}
$$

We can also compute the quantity $S^{\prime}$ defined in (20) which appears in the Smarr formula. It is given in terms of the horizon radius by

$$
S^{\prime}=\frac{\Omega_{D-2} r_{h}^{D-2}}{4(D-1) G} \sum_{k=0}^{k_{\max }} \frac{2 k(k-1) \hat{b}_{k}}{(D-2 k) r_{h}^{2(k-1)}} .
$$

Our goal in this section has been to develop expressions for the thermodynamic properties of AdS-Lovelock black holes purely in terms of the horizon radius. The final element of the Smarr relation (18) is the overall thermodynamic potential $\Theta$, which we will now compute. With this in hand, we can check all of our results are consistent with the Smarr relation. Substituting our result (31) for $c_{r}$ into the expression (30) for the $\theta^{(k)}$ and performing the sum over $k$ in (20) gives the result

$$
\Theta=-\frac{\Omega_{D-2} r_{h}^{D-3}}{8 \pi G(D-1)} \sum_{k=0}^{k_{\max }}\left(k-1+\frac{s_{(2)}}{s_{(1)}}\right) \frac{\hat{b}_{k}}{r_{h}^{2(k-1)}}
$$

Combining the results for the mass (32), surface gravity (36), entropy (40), $S^{\prime}$ in (41) and thermodynamic potential (42), it is now straightforward to check that the validity of the Smarr formula (18) for the AdS-Lovelock black holes. 


\section{$6 \quad$ Free energy and phase transitions}

In this section, we will make use of our results to give a general expression for the free energy of AdS-Lovelock black holes. We will discuss the behavior of this expression in the limits of large and small horizon radius, in which it is independent of the detailed choice of Lovelock couplings.

The free energy of asymptotically AdS black holes has been a topic of interest since the work of Hawking and Page [56] in Einstein gravity. They found that there exists a minimum temperature $T_{0}$ for such black holes which occurs for a horizon radius $r_{0}$. The temperature diverges both in the limit of large black holes and in the limit of zero horizon radius. Black holes with horizon $r_{h}<r_{0}$, like asymptotically flat black holes, have negative specific heat and cannot be in stable equilibrium with a thermal bath of radiation. However, solutions with $r_{h}>r_{0}$ have positive specific heat and can be in stable equilibrium. For large black holes with temperatures just above $T_{0}$, the free energy is positive and thermal AdS space, with zero free energy, represents that globally preferred thermodynamic state. However, the free energy of large black holes becomes negative above a critical temperature $T_{1}>T_{0}$ (or correspondingly for black holes with radii exceeding a certain threshold $r_{1}$ ) and the black hole is then the globally preferred state.

Hawking and Page found the free energy by computing the Euclidean action, which requires the analytic form of the AdS-Schwarzschild spacetimes. Similar computations of the free energy have been carried out in Gauss-Bonnet gravity [12, 43, 57, 58], where the explicit solutions are also known [10,11]. An expresssion for free energy in general Lovelock gravity is given in [59] which is calculated using a generalized quasi-local mass defined for spherically (plane or hyperbolic) symmetric spacetimes. However, theses computations are considerably more complicated. Our method offer a much a simpler route to the result and offers some degree of physical interpretation to the different terms in the result. It also yields the answer in the general Lovelock case, where do to the absence of explicit analytic solutions, computation of the Euclidean action may not be practical. Moreover as stated above, we envision further applications to even less well understood solutions, such as rotating Lovelock black holes.

It is worth noting explicitly that the equality of the free energy with the Euclidean action times the temperature continues to hold generally in Lovelock gravity theories. Hawking and Horowitz [60] used the Hamiltonian framework for Einstein gravity to demonstrate that $I_{E}=\beta M-S$ where $\beta$ is the Euclidean period. A similar construction works in Lovelock gravity, the basic steps being as follows.

Write the volume term of the Euclidean action for a static black hole in Hamiltonian variables, and then directly derive the boundary term for the action by varying this expression. The variation of the volume term is the same calculation that is done to find the mass (see 
[15, 20]) with an additional integration over Euclidean time. Evaluated on static solutions, the volume term of the action reduces to a sum of the constraints and hence vanishes. The value of the action is then given by the boundary terms. At infinity the boundary term is simply $M / T$. The Euclidean metric does not have a horizon. However, Hamiltonian evolution with respect to Euclidean time fails to be well defined at $r_{h}$. In order to compensate for this, one introduces an inner boundary at $r=r_{h}+\epsilon$. In the limit $\epsilon \rightarrow 0$ the boundary term is equal to the entropy $S$. One then has the result that the free energy defined by $F=T I_{E}$ coincides with the thermodynamic free energy $F=M-T S$. Note also that the free energy has been defined such that it vanishes for AdS6.

Let us see how the Hawking-Page transition arises in the present framework. In Lovelock gravity the cosmological constant is given by $\Lambda=-b_{0} / 2$, so that $b_{0}$ is positive for $\Lambda$ negative. Starting from $F=M-T S$, let us use the Smarr formula (18) to eliminate the mass. For Einstein gravity the quantities $s_{(2)}$ and $S^{\prime}=0$ in the Smarr relation vanish, while as shown in [19] the overall Lovelock thermodynamic potential is given by $\Theta=-V_{b h}$ where $V_{b h}$ is an effective volume for the black hole given by the flat (or AdS) spacetime volume of a sphere of radius $r_{h}$. One then arrives at an expression for the free energy

$$
\begin{aligned}
F & =\frac{1}{8 \pi G(D-3)}\left(\kappa A-b_{0} V_{b h}\right) \\
& =\frac{\Omega_{D-2} r_{h}^{D-2}}{8 \pi G(D-3)}\left(\kappa-\frac{b_{0} r_{h}}{(D-1)}\right)
\end{aligned}
$$

The important observation is that the apparent contribution of $\kappa A$ to the free energy has changed sign in (43) relative to the original expression $F=M-T S$. This is because the mass receives a positive contribution from $\kappa A$ in the Smarr formula and the overall net coefficient is always positive. For an asymptotically flat black hole, i.e. with vanishing $b_{0}$, this is the only term and the free energy is always positive. With a negative cosmological constant there is a negative definite contribution that takes the form of a cosmological pressure $b_{0}$ times an effective volume of the black hole $V_{b h}$. Hence the Hawking-Page phase transition at which the free energy changes sign may be thought of as arising from the $\Theta$ term in the Smarr relation, which is itself analogous to a $P V$-type contribution in classical thermodynamics.

To determine whether the positive or negative term dominates $F$ one needs to use know how the surface gravity behaves. Hawking and Page used the analytic solutions to compute $\kappa$ and found that the free energy is positive for small black holes as in Schwarzchild. However, they found that large black holes have negative free energy. Indeed, substituting $\kappa$ from equation (36) one recovers these results. However, rather than recall this case in more detail, we turn to an analysis of the free energy for general Lovelock black holes, which includes the case of Einstein gravity. We will see that the behavior of the free energy for large black holes is qualitatively the same as the Hawking-Page case. On the other hand,

\footnotetext{
${ }^{6}$ However, because of the presence of a mass gap for odd dimensional AdS-Lovelock black holes, the limit $r_{h} \rightarrow 0$ will not vanish in our subsequent expressions for $F$ given below.
} 
the free energy of small black holes differs between even and odd dimensions due to the alternating behavior of the surface gravity.

\subsection{Thermodynamic stability and phase transitions for Lovelock black holes}

There are a number of contributions to the general Lovelock free energy and the simplification made above in the Einstein case using the Smarr formula no longer yields an easily interpretable expression. Instead we simply substitute into the free energy the formulas for the mass (32) and the entropy (40) to obtain

$$
F=\frac{\Omega_{D-2} r_{h}^{D-3}}{16 \pi G(D-1)} \sum_{k=0}^{k_{\max }} \frac{\hat{b}_{k}}{r_{h}^{2(k-1)}}\left(1-\kappa r_{h} \frac{2 k}{(D-2 k)}\right)
$$

where $\kappa$ can also be regarded as in (36) as a function of the horizon radius and the Lovelock couplings. This expression for $F$ agrees with that of reference [12] in the case of GaussBonnet gravity and [59] in general Lovelock gravity, but disagrees] with that given in reference [43].

Let us start by examining the behavior of the free energy in the large black hole limit. Form (39), we see that the surface gravity grows like $b_{0} r_{h}$ for large $r_{h}$ in all dimensions. One then finds that there are both positive and negative contributions at leading order growing like $b_{0} r_{h}^{D-1}$. The net result for the free energy in the large black hole limit turns out to be negative,

$$
F \approx-\frac{\Omega_{D-2} b_{0} r_{h}^{D-1}}{16 \pi G(D-1)(D-2)}
$$

This result was also pointed out in [61] (see also [62, 63]) and is not surprising since the behavior in this regime is dominated by the cosmological constant and Einstein terms.

The behavior of the free energy (45) in the small black hole regime, on the other hand, is dominated by the highest curvature terms and differs between even and odd dimensions. In even dimensions both the mass $M$ and the product $\kappa S$ scale like $r_{h}$ in the limit of small horizon radius. One finds in this case that the positive contribution to the free energy coming from the mass dominates, giving

$$
F \approx \frac{\Omega_{D-2} \hat{b}_{k_{\max }} r_{h}}{32 \pi G(D-1)}, \quad D \text { even }
$$

\footnotetext{
${ }^{7}$ The expression for the free energy in [4] is obtained by computing the volume term in the Euclidean action, apparently without the inclusion of a boundary term. A subtraction of the action for pure $A d S$ at large radius is used to regularize the result. There are then several differences with our calculation. First, we implicitly use the Euclidean action with the boundary term that gives a well defined variational principal in the Hamiltonian variables. As described above the action on solutions is then given entirely by the boundary term and is equal to the finite quanity $M-T S$, without a need for regularization.
} 
In odd dimensions both the surface gravity and entropy vanish as $r_{h} \rightarrow 0$ (see equation (38)), while the mass has a finite positive limiting value, giving

$$
F \approx \frac{\Omega_{D-2} \hat{b}_{k_{\max }} r_{h}}{16 \pi G(D-1)}, \quad \text { Dodd }
$$

Hence $F$ is positive for small black holes in all dimensions. For $D$ even $F$ goes to zero, while for $D$ odd $F$ goes to a nonzero positive value.

It is also straightforward to compute the specific heat in the large and small black hole limits. Using the expressions for the mass and the surface gravity in section (5) we find for large black holes

$$
\frac{\partial M}{\partial T} \approx \frac{(D-2) \Omega_{D-2} r_{h}^{D-2}}{4 G}
$$

and for small black holes

$$
\begin{array}{ll}
\frac{\partial M}{\partial T} \approx-\frac{k_{\max } \Omega_{D-2} \hat{b}_{k_{\max }} r_{h}^{2}}{4 G(D-1)} & \text { D even } \\
\frac{\partial M}{\partial T} \approx \frac{k_{\max } \Omega_{D-2} \hat{b}_{\left(k_{\max }-1\right)}^{2} r_{h}}{4 G(D-1) \hat{b}_{k_{\max }}} & \text { D odd }
\end{array}
$$

where we have assumed that the couplings $b_{0}, b_{k_{\max }}$ and $b_{k_{\max }-1}$ are all nonzero.

Let us now summarize these results. While a detailed understanding of the global and local thermodynamic stability of AdS-Lovelock black holes throughout the entire range of horizon radii would require specifying the entire set of Lovelock couplings, the behavior in the large and small black hole regimes is generic. In dimensions, we find that the behavior of AdS-Lovelock black holes in these regimes is similar to that found by Hawking and Page in Einstein gravity [56]. Black holes become arbitrarily hot in both limits. Small black holes in even dimensions exhibit negative specific heat and have positive free energy, indicating instability to both perturbative and non-perturbative fluctuations. Large black holes, on the other hand, have positive specific heat and negative free energy, both indicating their stability to small thermal fluctuations and that they are the thermodynamically globally preferred state. If one assumes that the Lovelock couplings are such that the temperature stays positive for all $r_{h}$, then there must exist a minimum temperature $T_{0}$ below which no black hole solutions exist. As a consequence of these similarities, we can expect that even dimensional Lovelock black holes will at least have a simple Hawking-Page phase transition and possibly a more complicated structure of phase transitions, depending on the detailed behavior of the temperature and free energy over the whole range of horizon radii.

In odd dimensions only large black holes exist at very high temperatures. They have positive specific heat and negative free energy as in even dimensions and are therefore thermodynamically stable. The odd dimensional small black holes, on the other hand, have positive positive free energy and also have positive specific heat. So they are stable to 
small, but not large, thermal fluctuations, and unlike small black holes is even dimensions can be in stable equilibrium with a thermal bath.

In odd dimensions the low temperature picture is different. This was studied in $D=5$ Gauss-Bonnet gravity in references [12, 58]. Black holes exist for [12] temperatures down to $T=0$, and the low temperature black holes have positive specific heat, so there is a locally stable alternative to the gas state. At these low temperatures the pure gas state has lower free energy since $F_{\text {gas }} \rightarrow 0$ as $T \rightarrow 0$, while the black hole starts with $F$ of order $b_{k_{\max }}$. Still, a small black hole can exist in equilibrium with a low temperature gas, unlike the situation in even dimensions. So the behavior of small black holes in $D=5$ GaussBonnet gravity continues in odd dimensions as long as the highest curvature Lovelock term is included. For very high temperatures there is only the one large black hole state. As in even dimensions this is both globally and locally preferred.

\section{Discussion}

In this paper we started by deriving a fully explicit formula for the ADM mass of an asymptotically AdS spacetime in a generic Lovelock gravity theory, via the Hamiltonian methods of Regge \& Teitelboim [44. We then proceeded to study various thermodynamic properties of AdS-Lovelock black holes. In particular, we made use of the Killing-Lovelock potentials that exist in these spacetimes in order to evaluate the mass in terms of the horizon radius and the Lovelock couplings. After finding expressions for the surface gravity and entropy, these ingredients were assembled to give the free energy. All of these expressions are quite general, assuming only that solutions exist with the prescribed asymptotic forms.

As mentioned in the introduction, we envision further applications of these techniques to stationary solutions that are even "more unknown", such as higher dimensional rotating charged black holes, or rotating Lovelock black holes. Another possible direction for future work would be to look at black holes/branes in AdS with planar horizons. In this case the asymptotic boundary of a spatial slice is a plane rather than a sphere. If one of these directions is compact with length $L$, then it would be necessary to further extend the first law to include an appropriate $\delta L$ term as in the asymptotically flat Kaluza-Klein case [64].

\section{Acknowledgements}

The work of DK and JT was supported by NSF grant PHY-0555304. DK and JT also acknowledge the hospitality of the Centro de Ciencias de Benasque, Spain where this work was begun. The work of SR was funded by FONDECYT grant 3095018 and by the CON-

ICYT grant "Southern Theoretical Physics Laboratory" ACT-91. Centro de Estudios 
Científicos (CECS) is funded by the Chilean Government through the Millennium Science Initiative and the Centers of Excellence Base Financing Program of CONICYT. CECS is also supported by a group of private companies which at present includes Antofagasta Minerals, Arauco, Empresas CMPC, Indura, Naviera Ultragas and Telefónica del Sur. CIN is funded by CONICYT and the Gobierno Regional de Los Ríos.

\section{Appendix - Near horizon behavior of $g_{r r}$}

The horizon of a static black hole occurs where the norm of the static Killing field is zero. In the coordinates of the metric (11) this is at $r_{h}$ such that $\phi\left(r_{h}\right)=0$. In this appendix we show that if one assumes that the surface gravity is finite and that the Hamiltonian constraint equation is satisfied at $r_{h}$, then near the horizon it follows that $f(r) \sim \phi(r)$, and in particular that $f\left(r_{h}\right)=0, f^{\prime}\left(r_{h}\right)=2 \kappa$.

We point out that for the spherically symmetric black hole metric (11) it has been shown [11] [9] that the vacuum field equations imply that $-g_{t t}=1 / g_{r r}$, or $\phi(r)=f(r)$ everywhere. Clearly this is a stronger result than the result shown here. However, to derive the expressions for $M, \kappa$, and the $\Theta^{(k)}$ we only need to use the values of $f$ and $f^{\prime}$ at $r_{h}$, which can be found with correspondingly less work, as follows.

Assume that the metric function $\phi$ in (11) goes to zero like a power law as $r$ approaches $r_{h}$, i.e. that near the horizon

$$
\phi \simeq \phi_{1}\left(r-r_{h}\right)^{p}
$$

where $\phi_{1}$ is a constant. The surface gravity, given by $\kappa^{2}=-\frac{1}{2}\left(\nabla_{a} \xi_{b}\right) \nabla^{a} \xi^{b}$, then becomes

$$
\kappa^{2}=\frac{1}{2} p^{2} \lim _{r \rightarrow r_{h}}\left(r-r_{H}\right)^{p-2} f(r)
$$

In order for $\kappa^{2}$ to be finite, it must be that the metric function $f(r)$ behaves like

$$
f \simeq f_{1}\left(r-r_{h}\right)^{2-p}
$$

with $f_{1}$ another constant. Let us rewrite the expression (23) for $H_{\perp}^{(k)}$ as

$$
H^{(k)}=r^{1-2 k}\left[(D-2 k-1)(1-f) / r-k(1-f)^{k} f^{\prime}\right]
$$

and consider the constraint equation (24). If the power law index $p>1$, we see from line (A3) that $f^{\prime}$ diverges at the horizon, and inspection shows that $H=0$ cannot be satisfied at $r_{h}$. On the other hand, if the power law index $p<1$, then $f\left(r_{h}\right)=f^{\prime}\left(r_{h}\right)=0$ and again the constraint cannot be satisfied 8 at $r_{h}$. Hence, in order to have finite, non-zero surface

\footnotetext{
${ }^{8}$ If the spacetime is not vaccuum, the the gravitational constraint is $H=-16 \pi \rho$, and so powers $p<1$ are not ruled out.
} 
gravity, the power law index in (A2) and (A3) must be $p=1$, so that $\phi \sim \phi_{1}\left(r-r_{h}\right)$ and $f \sim f_{1}\left(r-r_{h}\right)$, which gives $\kappa^{2}=\frac{1}{2} \phi_{1} f_{1}$. We see that the overall numerical scale of $\kappa$ is not fixed by this argument, which makes sense because the normalization of $\kappa$ is fixed by the norm of the time-translation Killing vector at infinity, which requires knowing the function $\phi(r)$ throughout the spacetime. However, one can fix the scale by requiring that it gives the right answer for Schwarzchild.

\section{References}

[1] A. N. Aliev, "A Slowly rotating charged black hole in five dimensions," Mod. Phys. Lett. A 21, 751 (2006) [arXiv:gr-qc/0505003].

[2] A. N. Aliev, "Rotating black holes in higher dimensional Einstein-Maxwell gravity," Phys. Rev. D 74, 024011 (2006) arXiv:hep-th/0604207].

[3] H. -C. Kim, R. -G. Cai, "Slowly Rotating Charged Gauss-Bonnet Black holes in AdS Spaces," Phys. Rev. D77, 024045 (2008). [arXiv:0711.0885 [hep-th]].

[4] D. -C. Zou, Z. -Y. Yang, R. -H. Yue, "Thermodynamics of Slowly Rotating Charged Black Holes in anti-de Sitter Einstein-Gauss-Bonnet Gravity," Chin. Phys. Lett. 28, 020402 (2011). arXiv:1011.4348 [gr-qc]].

[5] R. Yue, D. Zou, T. Yu, P. Li and Z. Yang, "Slowly rotating charged black holes in anti-de Sitter third order Lovelock gravity," arXiv:1011.5293 [gr-qc].

[6] R. Emparan, T. Harmark, V. Niarchos, N. A. Obers, "World-Volume Effective Theory for Higher-Dimensional Black Holes," Phys. Rev. Lett. 102, 191301 (2009). arXiv:0902.0427 [hep-th]].

[7] R. Emparan, T. Harmark, V. Niarchos, N. A. Obers, "Essentials of Blackfold Dynamics," JHEP 1003, 063 (2010). arXiv:0910.1601 [hep-th]].

[8] M. M. Caldarelli, R. Emparan and B. Van Pol, "Higher-dimensional Rotating Charged Black Holes," JHEP 1104, 013 (2011) [arXiv:1012.4517 [hep-th]].

[9] J. T. Wheeler, "Symmetric Solutions To The Maximally Gauss-Bonnet Extended Einstein Equations," Nucl. Phys. B 273, 732 (1986).

[10] D. G. Boulware and S. Deser, "String Generated Gravity Models," Phys. Rev. Lett. 55, 2656 (1985).

[11] J. T. Wheeler, "Symmetric Solutions To The Gauss-Bonnet Extended Einstein Equations," Nucl. Phys. B 268, 737 (1986). 
[12] R. G. Cai, "Gauss-Bonnet black holes in AdS spaces," Phys. Rev. D 65, 084014 (2002) arXiv:hep-th/0109133.

[13] R. -G. Cai, Q. Guo, "Gauss-Bonnet black holes in dS spaces," Phys. Rev. D69, 104025 (2004). hep-th/0311020.

[14] R. -G. Cai, K. -S. Soh, "Topological black holes in the dimensionally continued gravity," Phys. Rev. D59, 044013 (1999). gr-qc/9808067].

[15] J. Crisostomo, R. Troncoso and J. Zanelli, "Black hole scan," Phys. Rev. D 62, 084013 (2000) arXiv:hep-th/0003271.

[16] A. Anabalon, N. Deruelle, Y. Morisawa, J. Oliva, M. Sasaki, D. Tempo, R. Troncoso, "Kerr-Schild ansatz in Einstein-Gauss-Bonnet gravity: An exact vacuum solution in five dimensions," Class. Quant. Grav. 26, 065002 (2009). arXiv:0812.3194 [hep-th]].

[17] A. Anabalon, N. Deruelle, D. Tempo, R. Troncoso, "Remarks on the Myers-Perry and Einstein Gauss-Bonnet Rotating Solutions," Int. J. Mod. Phys. D20, 639-647 (2011). arXiv:1009.3030 [gr-qc]].

[18] D. Kastor, "Komar Integrals in Higher (and Lower) Derivative Gravity," Class. Quant. Grav. 25, 175007 (2008) arXiv:0804.1832 [hep-th]].

[19] D. Kastor, S. Ray and J. Traschen, "Enthalpy and the Mechanics of AdS Black Holes," Class. Quant. Grav. 26, 195011 (2009) arXiv:0904.2765 [hep-th]].

[20] D. Kastor, S. Ray and J. Traschen, "Smarr Formula and an Extended First Law for Lovelock Gravity," arXiv:1005.5053 [hep-th].

[21] C. Teitelboim and J. Zanelli "Dimensionally Continued Topological Gravitation in Hamiltonian Form," Class. Quant. Grav. 4, L125 (1987); Also, "Constraint's theory and relativistic dynamics : proceedings," eds. G. Longhi and L. Lusanna (World Scientific, 1987).

[22] O. J. C. Dias, P. Figueras, R. Monteiro, J. E. Santos and R. Emparan, "Instability and new phases of higher-dimensional rotating black holes," Phys. Rev. D 80, 111701 (2009) arXiv:0907.2248 [hep-th]].

[23] M. M. Caldarelli, O. J. C. Dias, R. Emparan and D. Klemm, "Black Holes as Lumps of Fluid," JHEP 0904, 024 (2009) [arXiv:0811.2381 [hep-th]].

[24] O. Aharony, S. Minwalla and T. Wiseman, "Plasma-balls in large N gauge theories and localized black holes," Class. Quant. Grav. 23, 2171 (2006) arXiv:hep-th/0507219.

[25] S. Lahiri and S. Minwalla, "Plasmarings as dual black rings," JHEP 0805, 001 (2008) arXiv:0705.3404 [hep-th]]. 
[26] P. Kovtun, D. T. Son and A. O. Starinets, "Viscosity in strongly interacting quantum field theories from black hole physics," Phys. Rev. Lett. 94, 111601 (2005) arXiv:hep-th/0405231.

[27] Y. Kats and P. Petrov, "Effect of curvature squared corrections in AdS on the viscosity of the dual gauge theory," JHEP 0901, 044 (2009) [arXiv:0712.0743 [hep-th]].

[28] M. Brigante, H. Liu, R. C. Myers, S. Shenker and S. Yaida, "The Viscosity Bound and Causality Violation," Phys. Rev. Lett. 100, 191601 (2008) [arXiv:0802.3318 [hep-th]].

[29] X. H. Ge, Y. Matsuo, F. W. Shu, S. J. Sin and T. Tsukioka, "Viscosity Bound, Causality Violation and Instability with Stringy Correction and Charge," JHEP 0810, 009 (2008) arXiv:0808.2354 [hep-th]].

[30] X. H. Ge and S. J. Sin, "Shear viscosity, instability and the upper bound of the GaussBonnet coupling constant," JHEP 0905, 051 (2009) arXiv:0903.2527 [hep-th]].

[31] X. H. Ge, S. J. Sin, S. F. Wu and G. H. Yang, "Shear viscosity and instability from third order Lovelock gravity," Phys. Rev. D 80, 104019 (2009) arXiv:0905.2675 [hepth]].

[32] A. Buchel, J. Escobedo, R. C. Myers, M. F. Paulos, A. Sinha and M. Smolkin, "Holographic GB gravity in arbitrary dimensions," JHEP 1003, 111 (2010) arXiv:0911.4257 [hep-th]].

[33] A. Buchel and R. C. Myers, "Causality of Holographic Hydrodynamics," JHEP 0908, 016 (2009) arXiv:0906.2922 [hep-th]].

[34] J. de Boer, M. Kulaxizi and A. Parnachev, "Holographic Lovelock Gravities and Black Holes," JHEP 1006, 008 (2010) [arXiv:0912.1877 [hep-th]].

[35] D. M. Hofman, "Higher Derivative Gravity, Causality and Positivity of Energy in a UV complete QFT," Nucl. Phys. B823, 174-194 (2009). arXiv:0907.1625 [hep-th]].

[36] F. -W. Shu, "The Quantum Viscosity Bound In Lovelock Gravity," Phys. Lett. B685, 325-328 (2010). arXiv:0910.0607 [hep-th]].

[37] J. de Boer, M. Kulaxizi, A. Parnachev, "AdS(7)/CFT(6), Gauss-Bonnet Gravity, and Viscosity Bound," JHEP 1003, 087 (2010). [arXiv:0910.5347 [hep-th]].

[38] X. O. Camanho, J. D. Edelstein, "Causality constraints in AdS/CFT from conformal collider physics and Gauss-Bonnet gravity," JHEP 1004, 007 (2010). arXiv:0911.3160 [hep-th]].

[39] X. O. Camanho, J. D. Edelstein, "Causality in AdS/CFT and Lovelock theory," JHEP 1006, 099 (2010). [arXiv:0912.1944 [hep-th]]. 
[40] X. -H. Ge, B. Wang, S. -F. Wu, G. -H. Yang, "Analytical study on holographic superconductors in external magnetic field," JHEP 1008, 108 (2010). arXiv:1002.4901 [hep-th]].

[41] D. Lovelock, "The Einstein tensor and its generalizations," J. Math. Phys. 12, 498 (1971).

[42] T. Jacobson and R. C. Myers, "Black Hole Entropy And Higher Curvature Interactions," Phys. Rev. Lett. 70, 3684 (1993) arXiv:hep-th/9305016].

[43] M. Cvetic, S. Nojiri and S. D. Odintsov, "Black hole thermodynamics and negative entropy in deSitter and anti-deSitter Einstein-Gauss-Bonnet gravity," Nucl. Phys. B 628, 295 (2002) arXiv:hep-th/0112045].

[44] T. Regge and C. Teitelboim, "Role Of Surface Integrals In The Hamiltonian Formulation Of General Relativity," Annals Phys. 88, 286 (1974).

[45] L. F. Abbott and S. Deser, "Stability of Gravity with a Cosmological Constant," Nucl. Phys. B 195, 76 (1982).

[46] S. Deser, B. Tekin, "Gravitational energy in quadratic curvature gravities," Phys. Rev. Lett. 89, 101101 (2002). hep-th/0205318.

[47] S. Deser and B. Tekin, "Energy in generic higher curvature gravity theories," Phys. Rev. D 67, 084009 (2003) arXiv:hep-th/0212292.

[48] N. Deruelle, J. Katz, S. Ogushi, "Conserved charges in Einstein Gauss-Bonnet theory," Class. Quant. Grav. 21, 1971 (2004). gr-qc/0310098.

[49] J. Katz and G. Livshits, "Affine Gravity, Palatini Formalism and Charges", to appear in Gen. Rel. Grav., Festschrift in honor of Joshua Goldberg (2011).

[50] N. Okuyama, J. -i. Koga, "Asymptotically anti de Sitter spacetimes and conserved quantities in higher curvature gravitational theories," Phys. Rev. D71, 084009 (2005). [hep-th/0501044].

[51] A. Padilla, "Surface terms and the Gauss-Bonnet Hamiltonian," Class. Quant. Grav. 20, 3129 (2003) arXiv:gr-qc/0303082.

[52] R. G. Cai, "A Note on thermodynamics of black holes in Lovelock gravity," Phys. Lett. B 582, 237 (2004) |arXiv:hep-th/0311240].

[53] B. P. Dolan, "The cosmological constant and black-hole thermodynamic potentials," Class. Quant. Grav. 28, 125020 (2011). arXiv:1008.5023 [gr-qc]].

[54] M. Cvetic, G. W. Gibbons, D. Kubiznak, C. N. Pope, "Black Hole Enthalpy and an Entropy Inequality for the Thermodynamic Volume," arXiv:1012.2888 [hep-th]]. 
[55] R. C. Myers and J. Z. Simon, "Black Hole Thermodynamics in Lovelock Gravity," Phys. Rev. D 38, 2434 (1988).

[56] S. W. Hawking and D. N. Page, "Thermodynamics Of Black Holes In Anti-De Sitter Space," Commun. Math. Phys. 87, 577 (1983).

[57] S. Nojiri and S. D. Odintsov, "Anti-de Sitter black hole thermodynamics in higher derivative gravity and new confining-deconfining phases in dual CFT," Phys. Lett. B 521, 87 (2001) [Erratum-ibid. B 542, 301 (2002)] [arXiv:hep-th/0109122.

[58] Y. M. Cho and I. P. Neupane, "Anti-de Sitter black holes, thermal phase transition and holography in higher curvature gravity," Phys. Rev. D 66, 024044 (2002) arXiv:hep-th/0202140].

[59] H. Maeda, S. Willison and S. Ray, "Lovelock black holes with maximally symmetric horizons," arXiv:1103.4184 [gr-qc].

[60] S. W. Hawking and G. T. Horowitz, "The Gravitational Hamiltonian, action, entropy and surface terms," Class. Quant. Grav. 13, 1487 (1996) [arXiv:gr-qc/9501014].

[61] X. O. Camanho and J. D. Edelstein, "Higher curvature black holes," arXiv:1103.3669 [hep-th].

[62] R. -G. Cai, N. Ohta, "Black Holes in Pure Lovelock Gravities," Phys. Rev. D74, 064001 (2006). hep-th/0604088.

[63] R. -G. Cai, L. -M. Cao, N. Ohta, "Black Holes without Mass and Entropy in Lovelock Gravity," Phys. Rev. D81, 024018 (2010). arXiv:0911.0245 [hep-th]].

[64] D. Kastor, J. Traschen, "Stresses and Strains in the First Law for Kaluza-Klein Black Holes," JHEP 0609, 022 (2006). [hep-th/0607051]. 